in order to calculate the $P_{50}$ in these patients, before the results can be fully evaluated.

\section{Symposium III}

\section{Chromosomes and disease}

Leukaemia SYLVIA D. LAWLER (Department of Clinical Research, Royal Marsden Hospital, London) By using staining methods which produce characteristic banding patterns, it is now possible to identify precisely the individual chromosomes of the normal human set.

The components of structurally altered chromosomes can also be defined.For example, $O$ 'Riordan et al (1971) have shown that the Philadelphia chromosome, the deleted chromosome that is specifically associated with classical chronic myeloid leukaemia (CML), is a different member of the $\mathbf{G}$ group than the extra chromosome present constitutionally in Down's syndrome (Philadelphia chromosome $=$ no. 22 with a deletion involving the long arm. Down's syndrome $=$ trisomy 21 ).

Reeves et al (1972) have shown in four cases that the anomalous $F$ group chromosome found in a variable proportion of cells in about $20 \%$ of cases of polycythaemia vera (PV) is a number 20 , with a deletion involving the long arm, an observation which confirms the specificity of the anomaly in relation to $\mathbf{P V}$.

The metacentric 'marker' chromosome of $\mathrm{C}$ group size, found not infrequently in the blast transformation stage of CML, has now been identified as an iso-chromosome of the long arm of a number 17 (Lobb et al, 1972).

The identification of supernumerary or abnormal chromosomes is also proving useful in a follow-up study that is in progress in patients with acute leukaemia first examined in the untreated state. A chromosomal pattern is becoming apparent in acute lymphoblastic leukaemia (ALL). At diagnosis the cases can be divided into three classes according to the karyotypes of the bone marrow cells: (1) predominantly hyperdiploid cells, (2) occasionally hyperdiploid or pseudoploid and normal cells, (3) not hyperdiploid.

During the course of treatment in ALL the bone marrow does not convert immediately to cytogenetic normality. If serial samples of bone marrow are examined cases frequently are seen to go through a phase of having the occasional hyperdiploid cell before becoming normal. Such hyperdiploid cells usually do not have the samekaryotype as the original abnormal population. It remains to be seen whether relapse is associated with the reappearance of a population of cells that is chromosomally related to $\frac{0}{\vec{\sigma}}$ those found at diagnosis.

References

Lobb, D. S., Reeves, B. R., and Lawler, S. D. (1972). Lancet, 1, 849. 음 O'Riordan, M. L., Robinson, J. A., Buckton, K. E., and Evans, H. J. 드 (1971). Nature (Lond.), $230,167$. Reeves, B. R., Lobb, D. S., and Lawler, S. D. (1972). Humangenetik, $\frac{\bar{\Phi}}{\stackrel{\Phi}{1}}$
14, 159 .

Cancer of the Cervix A. I. SPRIGgs (Department of

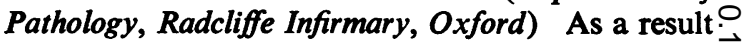
of cervical smears, a whole range of conditions is $\vec{\omega}$ available for study from normal through various $\stackrel{?}{?}$ presumptive precancerous stages up to fully devel- $\frac{\Omega}{8}$ oped invasive carcinoma of the cervix.

Established carcinoma shows chromosomal abnormalities comparable to those of other carcinomas; $\overrightarrow{.}$ there is a stemline with a karyotypediffering from the $\vec{\circ}$ normal in ways which are unique to the particular $\varnothing$ tumour, with gains and losses of chromosomes and ${ }^{\circ}$ often recognizable markers. Counts near to diploid 은 are the commonest, and counts above 92 are fairly rare. Most of the evidence favours the idea that the $z$ whole tumour originates from a single altered cell.

New karyotypically abnormal clones have also $\bar{z}$ been found in microcarcinoma (microinvasive $\underline{\text { Q }}$ carcinoma).

Carcinoma in situ and severe dysplasia present variations which are less easy to describe. All obs:servers have found aneuploidy, but there are cop flicting reports about the presence or absence of associated diploid cells. Cultures of epithelium have produced diploid cells only. The commonest regions in chromosome count distributions have been around 46 and around 80 . Sometimes the counts are con- $\overrightarrow{\vec{O}}$ sistent enough to suggest a clone. Similarity in the 3 karyotypes from two separate areas, sometimes with the additional evidence of markers, confirm that clonal proliferation has occurred. In other cases there is a wide spread of counts with no obvious stemline.

The mildest dysplasias have been shown in a few cases to have counts near to 46 , but even here $₹$ abnormal karyotypes have been described. Very few 0 analyses have been made from cells from normal cervices, and these have been found normal.

Taken together, the picture presented is of a tissue in which aneuploid cell lines compete for supremacy, or and in which a dominant clone finally selects itself. $N$ At what stage spontaneous regression or rejection $N$ of the lesion is still possible remains to be found out. Antenatal Diagnosis P. E. POLANI (Paediatric Research $\stackrel{\circ}{\complement}$ Unit, Guy's Hospital, London) Prenatal diagnosis $\stackrel{\Phi^{\circ}}{?}$ follows a number of different approaches directed at ${ }^{-}$ preventing or detecting fetal abnormality and, when 